$11-2020$

\title{
Childhood medulloblastoma
}

Naureen Mushtaq

Shahzadi Resham

Muhammad Shahzad Shamim

Bilal Mazhar Qureshi

Quratulain Riaz

See next page for additional authors

Follow this and additional works at: https://ecommons.aku.edu/pakistan_fhs_mc_med_haematol_oncol

Part of the Hematology Commons, Neurology Commons, Neurosurgery Commons, Oncology Commons, Pediatrics Commons, and the Surgery Commons 
Authors

Naureen Mushtaq, Shahzadi Resham, Muhammad Shahzad Shamim, Bilal Mazhar Qureshi, Quratulain Riaz, and Eric Bouffet 


\section{Childhood Medulloblastoma}

\section{NARRATIVE REVIEW}

Naureen Mushtaq ${ }^{1}$, Shahzadi Resham², Shahzad Shamim ${ }^{3}$, Bilal Mazhar Qureshi' ${ }^{4}$, Quratulain Riaz ${ }^{5}$, Eric Bouffet ${ }^{6}$

\begin{abstract}
Medulloblastoma is the most common malignant brain tumour in children and is a major cause of mortality and morbidity, particularly in low- and middle-income countries. It has been risk-stratified on the basis of clinical (age, metastasis and extent of resection) and histological subtypes (classic, desmoplastic and anaplastic). However, recently medulloblastoma has been sub-grouped by using a variety of different genomic approaches, such as gene expression profiling, micro-ribonucleic acid profiling and methylation array into 4 groups, namely Wingless, Sonic hedgehog, Group 3 and Group 4. This new sub-grouping has important therapeutic and prognostic implications. After acute leukaemia, brain tumour is the second most common malignancy in the paediatric age group. The improvement in outcome of acute lymphoblastic leukaemia in low- and middle-income countries reflects the relative simplicity of diagnostic procedures and management. Unlike leukaemia, the management of brain tumours requires a complex multidisciplinary approach, including neuro-radiologists, neurosurgeons with a paediatric expertise, neuropathologists, radiation oncologists and neuro-oncologists. In addition, the equipment required for the diagnosis (magnetic resonance imaging scan, histological, molecular and genetic techniques) and the management (operating room, radiation facilities) is a limiting factor in countries with limited resources. In Pakistan, there are very few centres able to treat children with brain tumours. The current literature review was planned to provide an update on the management of this tumour.
\end{abstract}

Keywords: Childhood brain tumours, Medulloblastoma, WNT, SHH.

\section{DOI: https://doi.org/10.5455/JPMA.293142}

\section{Introduction}

Childhood medulloblastoma is the most common malignant brain tumour. It arises in the posterior fossa and

1Department of Oncology, Aga Khan University Hospital, Karachi, Pakistan; 2Department of Pediatrics, Aga Khan University Hospital, Karachi, Pakistan; 3Department of Neurosurgery, Aga Khan University Hospital, Karachi, Pakistan; 4Department of Radiation Oncology. Aga Khan University Hospital, Karachi, Pakistan; ${ }^{5}$ Aga Khan University Hospital, Karachi, Pakistan; ${ }^{6}$ Department of Pediatric Oncology, The Hospital of Sick Children, Toronto, Canada.

Correspondence: Naureen Mushtaq. e-mail: naureen.mushtaq@aku.edu accounts for $20-25 \%$ of all primary paediatric central nervous system (CNS) tumours in high-income countries (HICs). ${ }^{1}$ The most common age of presentation in children is 3-10 years, and gender distribution has shown higher incidence in boys. However, the incidence from low-income countries (LICs) shows large variations ranging from $6.1 \%$ to $49.4 \%$ of reported series of paediatric brain tumours. This is likely due to multiple factors, including unavailability of proper data and lack of multidisciplinary teams even in tertiary care centres. ${ }^{2}$ It is also suspected that a number of cases are not diagnosed. ${ }^{2}$ Poor socioeconomic status, late diagnosis, and inaccessibility to treatment in most tertiary centres countrywide are major factors of poor prognosis. Although most countries with limited resources have neurosurgical facilities, radiation equipments and paediatric oncologic services, they often lack a multidisciplinary team approach that is critical in the management of this condition. ${ }^{3}$ There is a paucity of data regarding the incidence, treatment modalities and the outcome of children with brain tumours in low middleincome countries (LMICs). The current literature review was planned to provide recommendations for the treatment outcomes of childhood medulloblastoma in LMICs. ${ }^{3}$

\section{Methods}

Published literature was collected and summarised on the principles of multidisciplinary management of medulloblastoma from 2010 to 2017. PubMed was used as the resource database. A meta-narrative approach was used to identify and understand how the studies were conducted and conceptualised. Meta-narrative review has helped $\mathrm{n}$ the formulation of treatment guidelines for LMICs.

\section{Results}

Of the 65 articles on childhood medulloblastoma reviewed, $33(50.7 \%)$ were helpful in makng recommendations regarding diagnosis and treatment of the condition published in the last 5 years. Early presentation, recognition of signs and symptoms, availability of a formally trained physician, multidisciplinary approach and tumour board meetings in grouping with centres treating medulloblastoma in high-income settings will help standardise its clinical practice in LMICs. 


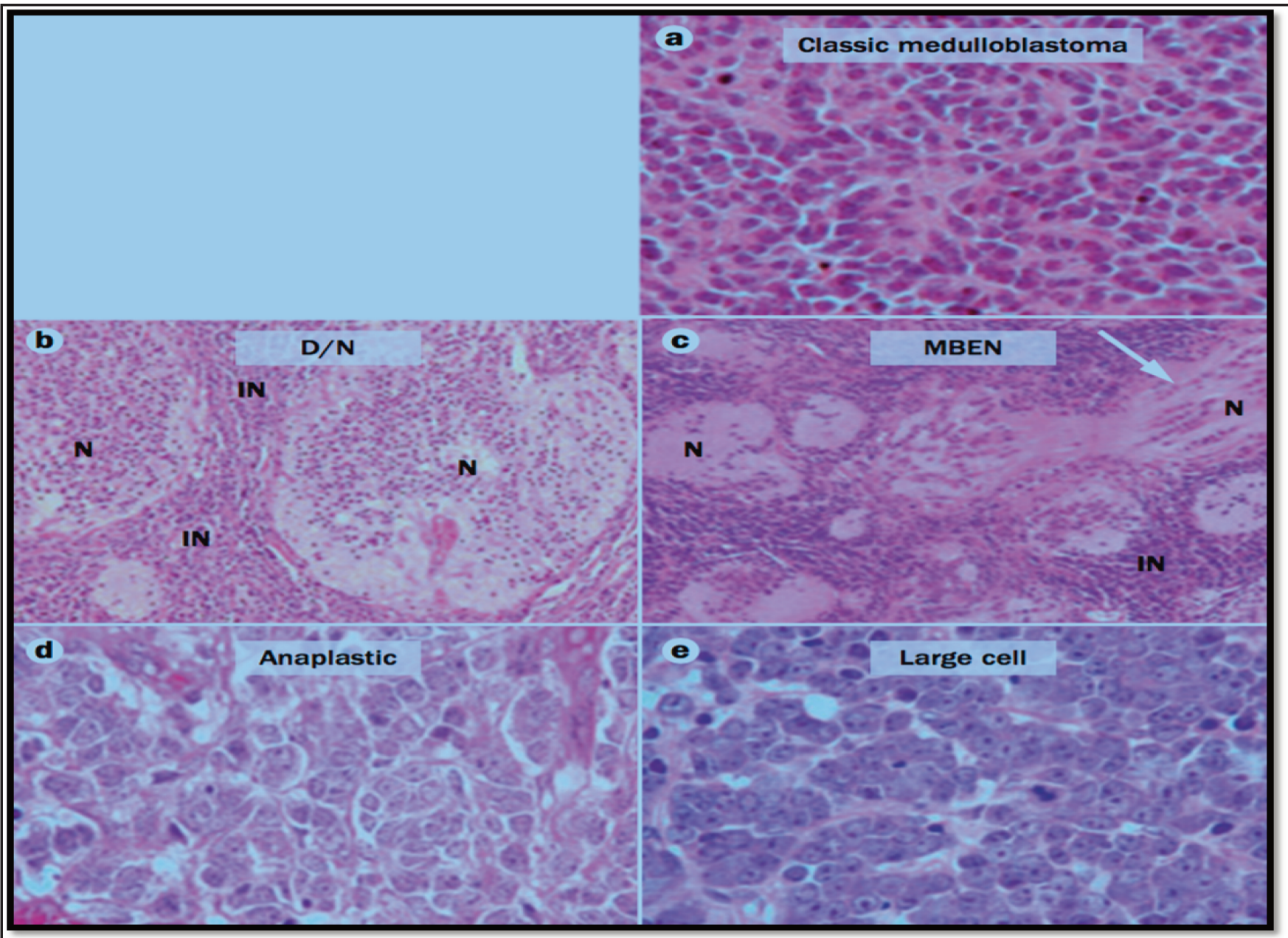

Figure: Histological variants of medulloblastoma. a) $\mid$ (lassic medulloblastoma,. b) $\mid$ D/N medulloblastoma c) |MBEN tissue. d) an anaplastic medulloblastoma. e) | a large cell medulloblastoma displaying the characteristic features of monomorphic cells with large nuclei and prominent nucleoli. (D/N, desmoplastic nodular; LCA, large cell/anaplastic; MBEN, medulloblastoma with extensive nodularity).

\section{Management Guidelines Histopathology}

Medulloblastoma is a heterogeneous disease on the basis of its various histological variants. Histologically, it is divided into the classic type which is the most common subtype; medulloblastoma with desmoplastic nodularity, which is more common in infants and young adults, medulloblastoma with extensive nodularity which is exclusively seen in infants, and anaplastic and/or large cell medulloblastoma. These four different histological subtypes of medulloblastoma can be grouped into two major pairs with many similar morphological features: nodular desmoplastic tumours comprising desmoplastic / nodular (D/N) and medulloblastoma with extensive nodularity (MBEN) variants; and anaplastic medulloblastoma overlapped with clear cell or large cell tumours (Figure). These variants have significant clinical utility on the management and outcomes: the desmoplastic variant has a better outcome than classic or anaplastic medulloblastomas in infants, and anaplastic medulloblastoma fares poorly compared to classic or desmoplastic tumours in both infants and children. ${ }^{4}$ Identification of the histological subtype before initiating treatment is critical. This will guide the oncologist to choose more intensive chemotherapy and higher dose of radiation for patients with higher risk, or, conversely, to consider standard or reduced dose radiation and maintenance chemotherapy for patients with low or standard risk, aiming to decrease treatment related morbidities.

\section{Presentation}

The most critical step in the management of medulloblastoma is to establish a correct diagnosis. Presenting sign and symptoms mainly depends on the site of involvement, like in any brain tumour. ${ }^{5}$ Most of these children present with signs of raised intracranial pressure due to the blockage of the aqueduct of Sylvius. They, 
therefore, often present with severe continuous morning headaches, recurrent projectile vomiting, neck pain and blurred vision. Cerebellar deficits which include gait problems and dysmetria can also be observed. As these presenting symptoms may be relatively non-specific, delay in diagnosis is frequent. In developing countries, we should keep our mind open for these symptoms as it will help us to diagnose these patients early and treat them in optimal conditions. ${ }^{3}$

\section{Molecular classification}

Over the last 10 years, large cooperative multi-institutional studies have been able to better characterise the biology of childhood medulloblastoma with the help of genomic and proteomic methods, mainly transcriptomic and methylomic analytical methods. As a result, medulloblastoma advanced genomic international consortium (MAGIC) described four principal molecular subgroups in medulloblastoma: Wingless, (WNT), Sonic hedgehog (SHH), Group 3, and Group 4. WNT and SHH tumours are marked by determining their specific signalling pathway leading to an up-regulation of the WNT and $\mathrm{SHH}$ pathways, respectively. The underlying genetic drivers or pathways associated with Groups 3 and 4 medulloblastoma are less identified, although some specific signatures have been described, like photoreceptors and those pertaining to or affecting the neurotransmitter gamma-aminobutyric acid (GABAergic) in Group 3, and neuronal and glutaminergic in Group 4. More studies are needed to better characterise these 2 subgroups.(6) When correlating clinical course with genetic subtyping, the most important implication of molecular classification in medulloblastoma is the prognosis, which differs markedly across tumour subgroups with WNT carrying the best outcome exceeding $90 \%$ with the current standard therapy. By contrast, patient with Group 3 tumours have the prognosis with survival rates in the range of $40-60 \%$. Other subtypes SHH and Group 4 have an intermediate event-free survival (EFS). The International Society of Neuropathology (ISN) in one of its consensus meetings recently recommended inclusion of molecular subgrouping and histology in risk stratification of medulloblastoma patients. 5

\section{Molecular sub-grouping WNTt subtype}

It is the least common (10-15\%), but the best studied subgroup. Children with this subtype have the best prognosis (>90\% survival) compared to other subtypes. Most patients present with localised (non-metastatic) disease. WNT bedulloblastoma exhibits the nuclear accumulation of $\beta$ catenin in more than $90 \%$ of the tumours. They further harbour mutations of CTNNB1 that encodes for $\beta$ catenin which explains the nuclear immunepositivity observed.6,7 Another hallmark of WNT medulloblastoma is the deletion of one copy of chromosome 6 . Both monosomy 6 and nuclear $\beta$ catenin accumulation are highly sensitive markers of this subtype. Histologically, most WNT tumours have classic histological features, but large cell/anaplastic (LCA) variants have been reported in literature as well.8 Most patients are children, particularly teenagers, and this subgroup is not seen in infants and adults. Gender distribution has shown a female predilection, unlike other subgroups which are more common in boys. These tumours are typically located in the cerebellar peduncle/ cerebellopontine angle cistern, and their resection can be challenging. However, as the prognosis is good, therefore it is recommended to treat with reduced dose of craniospinal irradiation (CSI) regardless of the extent of surgery, combined with conventional chemotherapy. The 5 -year overall survival is excellent. ${ }^{7}$

\section{SHH subtype}

SHH constitutes about $30 \%$ of all medulloblastoma. On presentation about $15-20 \%$ may have metastatic disease. Incidence is bimodal and this subtype affects infants and young adults more frequently. It is less common in children, where a specific variant associated with p53 mutation and aggressive behaviour can be observed. Sonic Hedgehog signalling pathways were first identified in patients with Gorlin Syndrome, characterised by a germline mutation of PTCH 1 (patched-1) tumour suppressor gene. SMO (smoothened) and SUFU (Suppressor of fused) mutations are the other genomic alterations associated with infantile $\mathrm{SHH}$. D/N histology is the most common histology associated with $\mathrm{SHH}$, but not all $\mathrm{SHH}$ exhibit $\mathrm{D} / \mathrm{N}$ architecture; literature also depicted some association with classic or LCA histology in SHH. Gender ratio revealed slight male preponderance. Cerebellar hemisphere is the most common site associated with $\mathrm{SHH}$ molecular subgroup. Five-year overall survival is $75 \%$, but the prognosis is extremely poor in patients with p53 mutation (mutant type) as it is commonly associated with Li-Fraumeni syndrome. 9,10

\section{Group 3 subgroup}

This subgroup accounts for $25 \%$ of all the medulloblastoma and a large proportion of patients with Group 3 tumours presents with metastatic disease at diagnosis. This subgroup is predominantly common in infants and preschoolers. Children with Group 3 and metastatic disease have the worst outcome with a 5 -year survival rate in the range of $50 \%$ or less. ${ }^{5}$ This subtype is not usually associated with any specific germline mutation; only few somatic genomic alterations MYC (MYC proto-oncogene) or OTX2 
(Orthodenticle homeobox-2) mutation in chromatin protein encoded by SMARCA4 (SWI/SNF Related, Matrix Associated, Actin Dependent Regulator Of Chromatin, Subfamily A, Member 4), KMT 2D (Histone-lysine Nmethyltransferase 2) and CHD 7 (Chromodomainhelicase-DNA-binding protein 7 ) have recently been identified. $6,8 \mathrm{As}$ far as the histology is concerned, Classic architecture and LCA are the two histological subtypes present in this subgroup. Males are affected twice as frequently then females. ${ }^{10,11}$

\section{Group 4 subgroup}

This is the most common subtype across the four subgroups (35\%), and is present in all age groups except in infants. 5 Males outnumber females. Children aged 5-10 years are most frequently affected (median age: 9 years). The prevalence of metastasis in Group 4 subgroup is 35\% at presentation. Although chromosome 17 abnormalities are not exclusive of Group 4, they are the hallmark of this subgroup. There is no specific germline mutation associated with Group 4. Literature showed KDM6A (lysine demethylase $6 \mathrm{~A}$ ) gene association in some of the Group 4 tumours that regulates the methylation of lysine 2.7 of histone H (H3 K27). ${ }^{12}$ Classic and LCA are 2 histological subtypes. Site of presentation includes midline and at the 4 th ventricle. The 5 -year overall survival is $75-85 \% .6,7$

\section{New Risk stratification}

Cooperative clinical groups and consortia have traditionally recognised three prognostic markers in childhood medulloblastoma: age at diagnosis, postoperative residual disease, and metastatic status. Patients aged $\leq 3$ years, leptomeningeal dissemination and postoperative residual tumour $\geq 1.5 \mathrm{~cm}^{2}$, are assigned to have high-risk disease. Children over 3-5 years of age with non-metastatic and fully resected tumour or having minimal residual disease $(<1.5$ $\mathrm{cm} 2$ ) post-operatively fall into the standard risk category, while children aged $<3$ years, those with metastatic disease and with a postoperative residue $>1.5 \mathrm{~cm}^{2}$ are considered high-risk. ${ }^{2}$ The clinical risk stratification, however, does not take into account the underlying biology of the disease and in particular the recent identification of 4 major subgroups. ${ }^{13}$ In 2015, a consensus meeting was held in Heidelberg, Germany, and data collected over the preceding decade was reviewed. A consensus was reached to further refine the risk stratification in the context of molecular subgrouping along with clinical features. An updated risk stratification has been then established for non-infant childhood medulloblastoma (ages 3-17). The most recent risk stratification incorporates molecular subtypes in risk groups and further classifies on the basis of prognosis (Table 1).14 Patients are identified as low-risk (>90\% survival), average/standard risk (70-90\% survival),
Table-1: Risk stratification on the basis of molecular sub-grouping.

\begin{tabular}{ll}
\hline Risk stratification & \multicolumn{1}{c}{ Molecular Sub-types } \\
\hline low-risk tumours & -Wingless (WNT)* subgroup \\
& - Non-metastatic subgroup Group 4 with \\
& - chromosome 11 loss or chromosome 17 gain \\
Standard risk & - SHH without MYCN* \\
& - SHH (p53 wild type) \\
& - Group 3 non metastatic a \\
& - Group 4 non metastatic \\
& - with no loss of chromosome 11 \\
High-risk group & - Metastatic disease with \\
& - SHH* or \\
& - Group 4 molecular variant or \\
& - MYCN*-amplified SHH* medulloblastoma $^{*}$ \\
Very high-risk & - Metastatic disease in \\
& - Group 3 or SHH* with TP53 (mutant) tumors \\
\hline
\end{tabular}

*SHH; Sonic hedgehog; *MYCN; MYC proto-Oncogene; *WNT; Wingless

high-risk (50-75\% survival), and very high risk $(<50 \%$ survival). The challenge in future is to gradually develop new protocols adapted to this new classification and explore targeted therapeutic approaches that integrate the molecular subgrouping in clinical trials and helps to improve the morbidities related to the intensive treatment approaches of medulloblastoma. 7,14,15

\section{Imaging}

The best diagnostic tool for any brain tumour is the MRI without and with contrast, but, if not available, valuable information can be obtained from computed tomography (CT) scan of brain, which can identify the underlying problem and contribute to referring the patient to a tertiary care centre. On CT or MRI imaging, the tumour is predominantly solid, located in the posterior fossa tumour, commonly arising from the vermis and less commonly from the cerebellar hemisphere. ${ }^{16}$ Ideally, a spinal MRI should be done prior to surgery to detect metastatic disease. A recent study correlated MRI characteristics, including tumour location and enhancement pattern with specific molecular subgroups of medulloblastoma. On MR imaging WNT tumours usually arise in the cerebellopontine angle (CPA), and the SHH most commonly involves the cerebellar hemispheres, while Groupd 3 and 4 tumours are located in the midline, filling the 4th ventricle with more enhancement in Group 3 than in Group 4 which only shows minimal enhancement.7

\section{Initial management}

Childhood medulloblastoma usually presents with signs of raised intracranial pressure that require immediate intervention. This involves gross total resection of tumour or, if not possible, cerebrospinal fluid (CSF) diversion procedure (ventriculo-peritoneal shunt or ventriculostomy) 
followed by tumour resection. Pre-operative dexamethasone will help to reduce cerebral oedema. Optimal management of medulloblastoma is multidisciplinary, therefore early involvement of all the clinicians, particularly neurosurgeon, pathologist, radiation oncologist and paediatric neuro-oncologist, is critical as soon as the child is admitted in a tertiary care centre. Final decisions for management should be made preferably after review by the multidisciplinary team (MDT). Due to the complexity of the management and subsequent follow-up, in most places, the paediatric oncologist is the designated person who chairs the team. There should be a contact person either from neurosurgery or paediatric oncology to support patients and families at all stages of illness. ${ }^{17}$

\section{Surgical guidelines}

Gross total resection remains the first-line treatment for medulloblastoma confined to the posterior fossa. The management of patients with extensive metastatic disease is more controversial. Advanced operative equipment, better understanding of tumour biology and posterior fossa anatomy and refined microsurgical techniques have made it possible for neurosurgeons to plan gross total resection in the majority of medulloblastoma cases, which significantly helps to risk-stratify these patients in average risk group, therefore improve their functional outcomes, by using a lower dose of CSI. However, surgery should aim at minimal morbidity, particularly when the tumour is adherent to brainstem or cranial nerves. With attempts at radical resections, the operative morbidity does increase, in particular with a higher incidence of posterior fossa mutism, but in experienced hands, the operative mortality is still less than 1 per cent. The extent of tumour resection has been shown to be better and the morbidity lower when the surgery is performed by specialised paediatric neurosurgeons, rather than by general neurosurgeons. ${ }^{17}$ Although gross surgical resection should remain the standard of care, further resection of metastatic deposits is not recommended when the risk of neurological morbidity is high. Second-look surgery is always an option if there is a large residual disease, which can be resected without significant neurological morbidity. CSF cytology (collected from a lumbar puncture at least 14 days after surgery, but preferable before surgery) should be analysed to complete the metastatic workup. ${ }^{18}$ Perioperative steroids (dexamethasone) help to reduce intracranial pressure. CSF diversion with an extra-ventricular drain (EVD) can be helpful in alleviating the hydrocephalus. However, insertion of a ventriculoperitoneal shunt prior to tumour resection is no longer recommended as the majority of patients will not need a shunt following tumour resection. The standard approach is through a midline sub-occipital craniectomy by making a surgical corridor through the natural space in between the cerebellar tonsils. Most of the medulloblastoma tumours are soft and are easily suckable. Every attempt should be made to limit the cerebellar retraction but for superiorly extending tumours, or those extending to the foramina of Luschka and Magendie, the cerebellar vermis may require to be split, in which case the inferior vermis is divided, sparing the superior vermis. Even for large tumours, despite their size, excellent grey-white differentiation allows an easy resection, even from the foramina and the floor of fourth ventricle. Tumour adherent to the floor should be dealt with with micro-dissection but in case of difficulty, it is acceptable for some tumour to be left behind. Intra-operative adjuncts, such as high magnification microscope, CUSA (cavitron ultrasonic surgical aspirator), high-quality bipolar and brainstem monitoring, are all useful. Postoperatively, 20-30\% of children may eventually require a CSF diversion, either with a third ventriculostomy or a ventriculoperitoneal shunt. ${ }^{19}$ Although some reports have pointed out the risk of shuntassociated seeding, this occurrence is exceedingly low and mostly seen when no chemotherapy is given. The postoperative MRI should be carried out within 48 hours, as post-operative changes will eventually interfere with contrast enhancement and make interpretation of the findings difficult. Apart from the risk of hydrocephalus, post-operative complications include haemorrhage usually from residual tumour, cerebellar swelling, new onset neurological deficits, including those related to brainstem swelling or injury, post-operative mutism, infection, etc. Posterior fossa syndrome, also known as cerebellar mutism syndrome, is associated with various signs and symptoms including mutism, swallowing disturbances, cranial nerves deficits, severe ataxia, decreased motor movement and emotional lability. Recent follow-up studies have shown that this complication has a serious impact on the neurocognitive outcome of affected patients. 3,17

\section{Radiotherapy guidelines}

Irradiation to the craniospinal axis with boost to the primary tumour bed is currently the recommended postoperative treatment for patients with average-risk medulloblastoma. CSI with whole posterior fossa boost is still the standard treatment for high-risk patients. Radiotherapy should be started as soon as possible and not later than 6 weeks after surgery as per International Society of Paediatric Oncology (SIOP) recommendations. ${ }^{3}$ There is evidence that delaying radiation treatment increases the risk of relapse. ${ }^{20}$ Considering the known problems of radiation waiting lists, late referrals and logistic issues in most countries with limited resources, the SIOP Paediatric Oncology in Developing Countries (PODC) adapted treatment recommendations for standard-risk medulloblastoma recommends doses used for the 
management of high-risk medulloblastoma if the radiation treatment starts after 7 weeks of tumour resection, regardless of the risk group. 21 However, this alternative has a cost for the patient, as reduced dose or radiation from 36 Gy to 23.4 Gy to the craniospinal axis in patients with standard-risk disease has shown decreased neurocognitive and endocrine late effects without compromising their progression-free survival.22 Radiation therapy for medulloblastoma comprises irradiation of the craniospinal axis, including the whole of brain, spinal cord and the complete area of CSF flow. The field should cover the complete thecal sac, including the exits of nerves at intervertebral foramina for meningeal coverage, and the cribriform plate. Three-dimensional conformal radiation treatment (3D-CRT) is preferred over the conventional twodimensional planning, but it might not be available at all centres. The initial craniospinal irradiation includes two lateral fields to cover the whole brain. These fields are matched with the posterior spinal field. The spine is covered caudally till the junction of second and third sacral vertebrae. A second spinal field is introduced for taller children to cover the whole area of interest. ${ }^{18}$ Previously considered the standard of care, the boost to the posterior fossa has now been abandoned for a more focal boost to the tumour bed in patients with non-metastatic, completely resected medulloblastoma. This boost is delivered with a $1-2 \mathrm{~cm}$ margin using 3D-CRT. Intensitymodulated radiation therapy (IMRT) may prove to be beneficial for certain cases, but is not widely available in the country. Boost can be done on the basis of bony anatomy if 3D-CRT is not available, but the doses of organ at risk like cochlea, cannot be ascertained.3,23 Total dose of radiation depends upon the risk stratification done preoperatively. The recommended dose per fraction is $180 \mathrm{cGy}$ with 5-6 fractions per week. Hyper fractionation $(2 \times 1 \mathrm{~Gy}$ or 2 x $1.3 \mathrm{~Gy} /$ day) has been used both in average-risk and high-risk patients. However, this technique has not shown any evidence of benefit. Standard-risk patients are treated with $2340 \mathrm{cGy}$ in 13 fractions to the craniospinal axis followed by focal local radiation to the tumour bed of the posterior fossa and total dose of 5400-5580 cGy in 17-18 more fractions. High-risk patients are prescribed CSI dose being 3600 cGy in 20 fractions followed by boost of 1980 cGy to the whole posterior fossa. Standard-risk patients who do not receive standard systemic therapy should be treated like high-risk patients with a higher CSI dose of 3600 cGy.23,24 The boost to the tumour bed may also apply to high-risk patients who do not show evidence of metastatic disease (i.e. patients with residual $>1.5 \mathrm{~cm}$ or patients who start radiation late). 25,26

Planning radiation to the craniospinal axis is a complex process and treatment at specialised centres having peer- reviewed practices is required for better outcome. ${ }^{23}$ This does not only include availability of necessary equipment but also trained and experience physicist, radiation oncologist and radiation therapy technologist for careful delivery of daily treatment. ${ }^{24}$ Quality assurance at each step of planning and delivery of this specialised treatment needs to be implemented. Correct daily positioning, placement of shielding relevant to the bony anatomy and matching the junction between cranial and spinal field and both spinal fields are only some of the many important concerns. ${ }^{23}$ Retrospective studies and prospective clinical trials have suggested that patients treated at major centres have a better outcome. 27,28

\section{Chemotherapy}

Different combinations of chemotherapeutic regimens have been shown to benefit the management of medulloblastoma. The most commonly used chemotherapy for patients $>3$ years of age with averagerisk disease consists of concomitant weekly vincristine along with radiotherapy followed by chemotherapeutic agents, including CCNU (Lomustine), cisplatin and vincristine, or cyclophosphamide, cisplatin, and vincristine. ${ }^{17}$ With such combination approaches, EFS is over $80 \%$ in patients with average-risk medulloblastoma. 26 The randomisation between cyclophosphamide and lomustine in combination with cisplatine and vincristine during maintenance did not show any difference in survival, although incidence of febrile neutropenia with cyclophosphamide was reported. This management regimen has been successfully employed in various LMICs and excellent survival was achieved for standard-risk patients. $^{3}$ Children $>3$ years, with high-risk medulloblastoma are treated with intensive chemotherapy during and post-radiation therapy. ${ }^{29}$ Pilot studies using daily etoposide or a combination vincristine and carboplatin during radiation, followed by maintenance chemotherapy have been tested with promising results and acceptable toxicity profile. ${ }^{30}$ Five-year EFS up to $70 \%$ was reported with these approaches. However, the survival benefit of such additions is still unknown. The results of ACNS0332, a randomised study comparing craniospinal irradiation with weekly vincristine administration and an experimental arm with the addition of a daily infusion of carboplatin as radiosensitiser are pending. A protocol using craniospinal irradiation followed by an intensified cisplatin, cyclophosphamide, vincristine, and etoposide regimen, supported by peripheral stem cell rescue, has shown similar outcomes. 26,27 With the shortage of radiation equipments and the difficulty to start radiotherapy in a timely manner, some physicians in countries with limited resources may consider the alternative of pre-radiation chemotherapy. There is evidence that this option has a negative impact on 
survival. The The GPOH HIT-91 (German Society of Pediatric Hematology and Oncology-Multicenter trial) randomised pre-radiation "sandwich" intensive chemotherapy, versus immediate radiotherapy with concomitant weekly vincristine, followed by maintenance chemotherapy in both arms, with vincristine, cisplatin and CCNU (Lomustine). The "sandwich" chemotherapy group was reported to have a higher incidence of severe neutropenia resulting in subsequent radiotherapy interruptions and decreased EFS. Some studies have also highlighted a high incidence of disease progression on pre-radiation chemotherapy. Therefore, it is a worldwide recommendation to consider radiation upfront. There is an ongoing debate regarding the benefit of weekly administration of vincristine during radiotherapy and its real impact on outcomes, particularly in teenagers who can develop sensory neuropathies with this approach. ${ }^{3}$ Many centres have also used varying low-intensity regimes with some success depending on the level of clinical facilities they may have. A Taiwanese study evaluated the results of six cycles of cisplatin $20 \mathrm{mg} / \mathrm{m}^{2}$ and etoposide $40-60$ $\mathrm{mg} / \mathrm{m}^{2}$ for 5 days and reported an 8-year EFS of $65 \% .30 \mathrm{Six}$ cycles of carboplatin at a dose of $500-600 \mathrm{mg} / \mathrm{m}^{2} / \mathrm{cycle}$, alternating with six cycles of cyclophosphamide $750 \mathrm{mg} / \mathrm{m}^{2}$, with vincristine has been used by an Egyptian group, and both achieved an EFS of $70 \%$. A regimen consisting of cycles of cisplatin at $90 \mathrm{mg} / \mathrm{m}^{2}$ with etoposide $150 \mathrm{mg} / \mathrm{m}^{2}$ for 2 days alternating with cycles of vincristine $2 \mathrm{mg} / \mathrm{m}^{2}$ and cyclophosphamide $1 \mathrm{~g} / \mathrm{m}^{2}$ for 2 days for a total of 6 courses has been used with some success OS $44 \%$ by Canan et al by the Turkey group but the hematological toxicity of this protocol was significant. ${ }^{31}$

\section{Long-term sequelae and follow-up}

Survivors of CNS tumours in general experience late effects resulting from the impact of the tumour itself and subsequent therapies, including neurosurgery, radiotherapy and chemotherapy. Susceptibility of developing brain injury needs long-term surveillance following treatment. Although there have been major advances in the management of medulloblastoma in terms of surgery, radiation, chemotherapy and supportive care, the long-term toxicity of these treatments remains a significant challenge and, as survival increases, these late effects are becoming a major issue among long-term survivors. 32

The main long-term sequelae include the risk of neurocognitive impairment, which includes memory dysfunction, learning issues, slow processing speed, psychological and emotional dysfunction. Risk factors associated with neurocognitive deficits include young age, postoperative mutism and higher dose of radiotherapy.
Endocrinopathies are extremely common among survivors, as a result of craniospinal radiation. Fertility dysfunction may also be secondary to chemotherapy. The occurrence of secondary malignancies is not uncommon, in particular meningioma that can have a long latency period up to 30 years. All these treatment-related morbidities can result in moderate to severe disability and worsening of quality of life. These have major implications in adult life with reduced attainment of expected milestones, including education, employment and independent living. ${ }^{33}$ Regular follow-up visits are essential and should be emphasised. On every visit a thorough physical exam should be done to evaluate the morbidities of chemotherapy and radiation. These include anthropometric monitoring and basal metabolic index (BMI), examination of the skin in the radiation fields, a search for thyroid nodules, signs of cerebellar dysfunction, any visual field defects, hearing problems, peripheral neuropathies and spine for scoliosis. Previous spinal radiotherapy may affect spinal growth, induce scoliosis, or cause an increased risk of degenerative arthritis and osteoporosis; hence, early screening for osteoporosis is recommended and any physical work which involves lifting heavy weight should be avoided. ${ }^{9}$ A close collaboration with an endocrinologist is mostly needed as these patients are at high risk for growth hormone deficiency, ACTH (Adrenocorticotropic hormone) deficiency, hypothyroidism and metabolic syndrome. There is high risk of secondary tumours and therefore, high index of suspicion is needed for lesions in the radiotherapy field. These survivors should be advised to avoid sunburn and wearing a hat in bright sunlight. They should have audiometry every 1-2 years due to risk of hearing loss with platinum compounds. An important point of concern during the follow up visits is the mental health of the patient which is a combined effect of radiation and chemotherapy. Behaviour changes can be due to prolonged absence from school, prolonged hospitalisation, physical disabilities (short stature, obesity, alopecia, endocrinopathies), learning and psychosocial difficulties. The physician should enquire regarding schooling and education and behaviour with timely referral to psychology and social work team, if needed. These survivors are likely to benefit from family counselling, psychology and psychiatry consultation. 34

Important point of consideration is that most of these quality-of-life issues are closely related to the management of the tumour itself. Proper risk stratification of the tumour to identify patients that require high-dose radiation and chemotherapy from those that can be treated with less aggressive treatment on the basis of an appropriate staging and molecular testing can help improve the late effects of management. Safer radiation technique, like IMRT 
Table-2: Required Evaluations Following Completion of Protocol Therapy.

\begin{tabular}{|c|c|c|c|c|c|c|c|c|c|c|c|c|c|c|c|c|c|c|}
\hline Evaluation & 3 mon & 4 mon & $6 \mathrm{mon}$ & $8 \mathrm{mon}$ & 9 mon & $12 \mathrm{mon}$ & 15 mon & $16 \mathrm{mon}$ & $18 \mathrm{mon}$ & 20 mon & $21 \mathrm{mon}$ & 2 yrs & $2.5 \mathrm{yrs}$ & 3 yrs & $3.5 \mathrm{yrs}$ & 4 yrs & Annually & $\begin{array}{l}\text { At Relapse } \\
\text { Disease } \\
\text { Progression }\end{array}$ \\
\hline $\begin{array}{l}\text { History, Physical with } \\
\text { Neurologic Exam }\end{array}$ & $x$ & $x$ & $x$ & & $x$ & $x$ & $x$ & & $x$ & & $x$ & $x$ & $x$ & $x$ & $X$ & $x$ & $x$ & $x$ \\
\hline $\begin{array}{l}\text { Liver Function, BUN, } \\
\text { Creatinine, Electrolytes } \\
(\mathrm{Ca}, \mathrm{Mg}), \mathrm{CBC}\end{array}$ & $X$ & $x$ & $X$ & & $x$ & $x$ & $x$ & & $x$ & & $x$ & $x$ & $x$ & $x$ & $X$ & $X$ & $X$ & \\
\hline $\begin{array}{l}\text { MRI of head } \\
\text { (with contrast) }\end{array}$ & $x$ & $x$ & $x$ & & $x$ & $x$ & $x$ & & $x$ & & $x$ & $x$ & $x$ & $x$ & $x$ & $x$ & $x$ & $x$ \\
\hline $\begin{array}{l}\text { Spinal MRI } \\
\text { (with contrast)‡ }\end{array}$ & $X \$$ & $X \$$ & $X \$$ & $X \$$ & $X \$$ & $X \$$ & $X \$$ & & $X \$$ & & $X \$$ & $X \$$ & $X \$$ & $X \$$ & $X \$$ & $X \$$ & $X \$$ & $x$ \\
\hline $\begin{array}{l}\text { Lumbar CSF Cytology } \\
\text { Audiogram }\end{array}$ & & $X \$$ & $x$ & $X \$$ & & $\begin{array}{c}X \$ \\
X\end{array}$ & & $X \$$ & $x$ & $X \$$ & & $\begin{array}{c}X \$ \\
X\end{array}$ & $X \$$ & $\begin{array}{l}X \$ \\
X\end{array}$ & & $\begin{array}{l}X \$ \\
X\end{array}$ & $\begin{array}{l}X \$ \\
X\end{array}$ & $x$ \\
\hline $\begin{array}{l}\text { Thyroid Function Evaluati } \\
\text { (Free T4 and TSH) }\end{array}$ & & & $x$ & & & $X$ & & & & & & $X$ & & $x$ & & $X$ & $X$ & \\
\hline $\begin{array}{l}\text { LH FSH, estradiol or } \\
\text { testosterone }\end{array}$ & & & & & & $x$ & & & & & & $x$ & & $x$ & & $x$ & $x$ & \\
\hline $\begin{array}{l}\text { Serial Measurement of } \\
\text { Height (Stature)\% }\end{array}$ & & & $x$ & & & $x$ & & & & & & $x$ & & $x$ & & $x$ & $x$ & \\
\hline
\end{tabular}

‡:Spinal MRI should include complete spine (cervical, thoracic, lumbar and sacral); \%:Refer to endocrinologist if a growth below the 3rd percentile, drop in height percentile on growth grid, velocity $<4-5 \mathrm{~cm} /$ year in childhood or lack of pubertal growth spurt; $\$$ Obtain if initially positive. Patients with M1-M3 disease require the more frequent scans.

(Intensity modulated radiation therapy) may be used where required.

Observations required at the end of therapy to monitor the long-term sequelae are critical (Table 2).35

\section{Discussion}

In HICs, individualisation of therapy for children with medulloblastoma represents a major goal. In recent years, histopathological and molecular disease features have been identified with the potential to provide a more refined stratification of disease. 26 Unfortunately, these specialised investigations are still not available in most centres dealing with paediatric brain tumours in LMICs, especially in Pakistan. Hence, risk stratification at the time of diagnosis still remains elusive.

Limited access to supportive care, poor baseline nutritional status, concomitant infections or co-morbidities, and unavailability of rehabilitation service, have a significant influence in the treatment choices and outcomes in the developing world. Therefore, guidelines and treatment protocols developed in HICs may not be applicable in our part of the world. The PODC committee of SIOP neurooncology Working Group has, therefore, developed guidelines for LMICs for the treatment of children with standard-risk medulloblastoma. ${ }^{3}$ Another working PODC group, with members of the Paediatric Oncology Radiation society (PROS) have also developed adapted guidelines for radiation in $\mathrm{LMICs.}{ }^{22}$

These recommendations provide the opportunity to develop a common strategy for the management of medulloblastoma patients across Pakistan. Consistency in the management between different centres will offer a unique opportunity to collect national data and measure the gap between Pakistan and other countries or cooperative groups.

One should keep in mind the complexity of the management of this condition and the need to have a perfect and timely interaction between different team members. When a patient is initially seen in a neurosurgical unit without a multidisciplinary paediatric neuro-oncology programme, early referrals is critical and a peer review of every single patient with a multi-disciplinary approach will benefit us not only in maintaining a patient log, but also help in the establishment of evidence-based guidelines based on the response of therapy.

\section{Conclusion}

Despite the recent integration of biological markers in the management of medulloblastoma, some questions still need to be answered as to how we can incorporate these molecular markers in LMICs where resources are scarce and knowledge about this condition is still very limited. In this context, multidisciplinary team management is the key. Twinning between HICs and LMICs can improve the 
outcome in these children.

\section{Disclaimer: None. \\ Conflict of Interest: None. Source of Funding: None.}

\section{References}

1. Rutkowski S, von Hoff K, Emser A, Zwiener I, Pietsch T, FigarellaBranger D, et al. Survival and prognostic factors of early childhood medulloblastoma: an international meta-analysis. J Clin Oncol 2010; 28: 4961-8.

2. Riaz Q, Naeem E, Fadoo Z, Lohano M, Mushtaq N. Intracranial tumors in children: a 10-year review from a single tertiary health-care center. Childs Nerv Syst 2019; 35: 2347-53.

3. Parkes J, Hendricks $M$, Ssenyonga $P$, Mugamba J, Molyneux $E$, Schouten-van Meeteren A, et al. SIOP PODC adapted treatment recommendations for standard-risk medulloblastoma in low and middle income settings. Paediatr Blood Cancer 2015; 62: 553-64.

4. Louis DN, Perry A, Reifenberger G, von Deimling A, Figarella-Branger D, Cavenee WK, et al. The 2016 World Health Organization Classification of Tumours of the Central Nervous System: a summary. Acta Neuropathol 2016; 131: 803-20.

5. Taylor MD, Northcott PA, Korshunov A, Remke M, Cho YJ, Clifford SC, et al. Molecular subgroups of medulloblastoma: the current consensus. Acta Neuropathol 2012; 123: 465-72.

6. Kool M, Korshunov A, Remke M, Jones DT, Schlanstein M, Northcott PA, et al. Molecular subgroups of medulloblastoma: an international meta-analysis of transcriptome, genetic aberrations, and clinical data of WNT, SHH, Group 3, and Group 4 medulloblastomas. Acta Neuropathol 2012; 123: 473-84.

7. Iv M, Zhou M, Shpanskaya K, Perreault S, Wang Z, Tranvinh E, et al. MR Imaging-Based Radiomic Signatures of Distinct Molecular Subgroups of Medulloblastoma. AJNR Am J Neuroradiol 2019; 40: 154 61

8. Northcott PA, Korshunov A, Pfister SM, Taylor MD. The clinical implications of medulloblastoma subgroups. Nat Rev Neurol 2012; 8: 340 51.

9. Cavalli FMG, Remke M, Rampasek L, Peacock J, Shih DJH, Luu B, et al. Intertumoural Heterogeneity within Medulloblastoma Subgroups. Cancer Cell 2017; 31: 737-54.

10. Kawauchi D, Robinson G, Uziel T, Gibson P, Rehg J, Gao C, et al. A mouse model of the most aggressive subgroup of human medulloblastoma. Cancer Cell 2012; 21: 168-80.

11. Schwalbe EC, Lindsey JC, Nakjang S, Crosier S, Smith AJ, Hicks D, et al. Novel molecular subgroups for clinical classification and outcome prediction in childhood medulloblastoma: a cohort study. Lancet Oncol 2017;18: 958-71.

12. Pugh TJ, Weeraratne SD, Archer TC, Pomeranz Krummel DA, Auclair $D$, Bochicchio J, et al. Medulloblastoma exome sequencing uncovers subtype-specific somatic mutations. Nature 2012; 488: 106-10.

13. Coluccia D, Figuereido C, Isik S, Smith C, Rutka JT. Medulloblastoma: Tumour Biology and Relevance to Treatment and Prognosis Paradigm. Curr Neurol Neurosci Rep 2016; 16: 43.

14. Ramaswamy V, Remke M, Bouffet E, Bailey S, Clifford SC, Doz F, et al. Risk stratification of childhood medulloblastoma in the molecular era: the current consensus. Acta Neuropathol 2016; 131: 821-31.

15. Schwalbe EC, Williamson D, Lindsey JC, Hamilton D, Ryan SL, Megahed $\mathrm{H}$, et al. DNA methylation profiling of medulloblastoma allows robust subclassification and improved outcome prediction using formalin-fixed biopsies. Acta Neuropathol 2013; 125: 359-71.

16. Yeom KW, Mobley BC, Lober RM, Andre JB, Partap S, Vogel H, et al. Distinctive MRI features of paediatric medulloblastoma subtypes. AJR Am J Roentgenol 2013; 200: 895-903.
17. Fischer C, Petriccione M, Donzelli M, Pottenger E. Improving Care in Pediatric Neuro-oncology Patients: An Overview of the Unique Needs of Children With Brain Tumors. J Child Neurol 2016; 31: 488505.

18. Thompson EM, Hielscher T, Bouffet E, Remke M, Luu B, Gururangan $S$, et al. Prognostic value of medulloblastoma extent of resection after accounting for molecular subgroup: a retrospective integrated clinical and molecular analysis. Lancet Oncol 2016; 17: 484-95.

19. Nigim F, Critchlow JF, Kasper EM. Role of ventriculoperitoneal shunting in patients with neoplasms of the central nervous system: An analysis of 59 cases. Mol Clin Oncol 2015; 3: 1381-6.

20. Lannering B, Rutkowski S, Doz F, Pizer B, Gustafsson G, Navajas A, et al. Hyperfractionated versus conventional radiotherapy followed by chemotherapy in standard-risk medulloblastoma: results from the randomized multicenter HIT-SIOP PNET 4 trial. J Clin Oncol 2012; 30: 3187-93.

21. Ahern V, Koh ES, Gebski V, Sathiyaseelan Y. Paediatric medulloblastoma: Patterns of care and radiotherapy quality assurance in Australia. Aust Radiol 2007; 51: 458-64.

22. Conklin HM, Ashford JM, Clark KN, Martin-Elbahesh K, Hardy KK, Merchant TE, et al. Long-Term Efficacy of Computerized Cognitive Training Among Survivors of Childhood Cancer: A Single-Blind Randomized Controlled Trial. J Paediatr Psychol 2017; 42: 220-31.

23. Parkes J, Hess C, Burger H, Anacak Y, Ahern V, Howard SC, et al. Recommendations for the treatment of children with radiotherapy in low- and middle-income countries (LMIC): A position paper from the Paediatric Radiation Oncology Society (PROS-LMIC) and Paediatric Oncology in Developing Countries (PODC) working groups of the International Society of Paediatric Oncology (SIOP). Paediatr Blood Cancer 2017; 64: e26903.

24. Carrie C, Grill J, Figarella-Branger D, Bernier V, Padovani L, Habrand $\mathrm{J}$, et al. Online quality control, hyperfractionated radiotherapy alone and reduced boost volume for standard risk medulloblastoma: longterm results of MSFOP 98. J Clin Oncol 2009; 27: 1879-83.

25. Packer RJ, Zhou T, Holmes E, Vezina G, Gajjar A. Survival and secondary tumours in children with medulloblastoma receiving radiotherapy and adjuvant chemotherapy: results of Children's Oncology Group trial A9961. Neuro Oncol 2013; 15: 97-103.

26. Ramaswamy V, Remke M, Adamski J, Bartels U, Tabori U, Wang X, et al. Medulloblastoma subgroup-specific outcomes in irradiated children: who are the true high-risk patients? Neuro Oncol 2016; 18: 2917.

27. Danjoux CE, Jenkin RD, McLaughlin J, Grimard L, Gaspar LE, Rashid Dar A, et al. Childhood medulloblastoma in Ontario, 1977-1987: Population-based results. Med Pediatr Oncol 1996; 26: 1-9.

28. Tait DM, Thornton-Jones H, Bloom HJ, Lemerle J, Morris-Jones P. Adjuvant chemotherapy for medulloblastoma: the first multi-centre control trial of the International Society of Paediatric Oncology (SIOP I). Eur J Cancer 1990; 26: 464-9.

29. Lin CY, Erkek S, Tong Y, Yin L, Federation AJ, Zapatka M, et al. Active medulloblastoma enhancers reveal subgroup-specific cellular origins. Nature 2016; 530: 57-62.

30. Esbenshade AJ, Kocak M, Hershon L, Rousseau P, Decarie JC, Shaw S,et al: A Phase II feasibility study of oral etoposide given concurrently with radiotherapy followed by dose intensive adjuvant chemotherapy for children with newly diagnosed high-risk medulloblastoma (protocol POG 9631): A report from the Children's Oncology Group. Pediatr Blood Cancer 2017; 64: 10.

31. Akyuz C, Varan A, Kupeli S, Akalan N, Soylemezoglu F, Zorlu F, et al. Medulloblastoma in children: A 32-year experience from a single institution. J Neurooncol 2008; 90: 99-103

32. Riggs L, Bouffet E, Laughlin S, Laperriere N, Liu F, Skocic J, et al. Changes to memory structures in children treated for posterior fossa tumours. J Int Neuropsychol Soc 2014; 20: 168-80. 
33. Boman KK, Hornquist L, De Graaff L, Rickardsson J, Lannering B, Gustafsson G. Disability, body image and sports/physical activity in adult survivors of childhood CNS tumours: population-based outcomes from a cohort study. J Neurooncol 2013; 112: 99-106.

34. Riggs L, Piscione J, Laughlin S, Cunningham T, Timmons BW, Courneya KS, et al. Exercise training for neural recovery in a restricted sample of paediatric brain tumour survivors: a controlled clinical trial with crossover of training versus no training. Neuro Oncol 2017; 19: 440-50.

35. Michalski J, Clinical Oncology Group. A Study Evaluating Limited Tar-
getVolume Boost Irradiation 645 and Reduced Dose Craniospinal Radiotherapy (18.00 Gy) and Chemotherapy in 646 Children with Newly Diagnosed Standard Risk Medulloblastoma: A Phase III 647 DoubleRandomized Trial. Children Oncology Group. Oncology Group National Cancer Institute. [Online] 2013 [Cited 2020 January 21]. Available from: URL: https://clinicaltrials.gov/ct2/show/ NCT00085735 